\title{
Monitoring the decreasing trend of testicular cancer mortality in Spain during 2005-2019 through a Bayesian approach
}

\author{
Ramon Clèries a,b,*, José Miguel Martínez ${ }^{\mathrm{c}, \mathrm{d}}$, Josep Maria Escribà a , Laura Esteban ${ }^{\mathrm{a}}$, \\ Laura Pareja ${ }^{\mathrm{a}}$, Josep Maria Borràs ${ }^{\mathrm{a}, \mathrm{b}}$, Josepa Ribes ${ }^{\mathrm{a}, \mathrm{b}}$ \\ ${ }^{a}$ Catalan Plan for Oncology, Institut Català d'Oncologia - IDIBELL, Gran Via s/n, km 2,7 L'Hospitalet de Llobregat, Barcelona 08907, Spain \\ ${ }^{\mathrm{b}}$ Dept. of Clinical Sciences, Universitat de Barcelona, Barcelona, Spain \\ 'Centro de Investigación en Salud Laboral (CiSAL), Universitat Pompeu Fabra, Barcelona, Spain \\ ${ }^{\mathrm{d}}$ Grup de Recerca de Desigualtats en Salut (GREDS/EMCONET), Universitat Pompeu Fabra, Barcelona, Spain
}

A R T I C L E I N F O

Article history:

Accepted 4 March 2010

Keywords:

Testicular cancer

Mortality

Time trends

Bayesian

Projections

\begin{abstract}
A B S T R A C T
Purpose: To assess time trends of testicular cancer (TC) mortality in Spain for period 1985-2019 for age groups 15-74 years old through a Bayesian age-period-cohort (APC) analysis. Methods: A Bayesian agedrift model has been fitted to describe trends. Projections for 2005-2019 have been calculated by means of an autoregressive APC model. Prior precision for these parameters has been selected through evaluation of an adaptive precision parameter and 95\% credible intervals (95\% CRI) have been obtained for each model parameter. Results: A decrease of $-2.41 \%$ (95\% CRI: $-3.65 \% ;-1.13 \%)$ per year has been found for TC mortality rates in age groups 15-74 during 1985-2004, whereas mortality showed a lower annual decrease when data was restricted to age groups $15-54(-1.18 \%$; 95\% CRI: $-2.60 \%$; $-0.31 \%)$. During 2005-2019 is expected a decrease of TC mortality of $2.30 \%$ per year for men younger than 35 , whereas a leveling off for TC mortality rates is expected for men older than 35. Conclusions: A Bayesian approach should be recommended to describe and project time trends for those diseases with low number of cases. Through this model it has been assessed that management of TC and advances in therapy led to decreasing trend of TC mortality during the period 1985-2004, whereas a leveling off for these trends can be considered during 2005-2019 among men older than 35.
\end{abstract}

(C) 2010 Elsevier Ltd. All rights reserved.

\section{Introduction and purpose}

Testicular cancer (TC) is the most common cancer among men aged 15-44 in European population, where the lowest incidence and mortality rates from TC are found in Eastern and Southern European countries [1]. Based on the most updated estimates, Spain is the Southern European country with lowest incidence (1.9 per 100,000 men-years) and mortality rates $(0.1$ per 100,000 menyears), accounting for $1 \%$ of the cancers in Spanish men [1].

TC mortality had declined in the majority of European countries, whereas incidence had risen in all 12 European countries studied with highest increases were found in Slovenia and Spain [2,3]. Causes of TC are largely unknown with no satisfactory hypotheses to explain the trends, although evidence suggests that the process of carcinogenesis could be initiated perinatally [4] or early in life [5], perhaps even in utero [6]. TC is

\footnotetext{
* Corresponding author at: Catalan Plan for Oncology, Institut Català d'Oncologia - IDIBELL, Gran Via s/n, km 2,7 L'Hospitalet de Llobregat, Barcelona 08907, Spain. Tel.: +34 9326078 12; fax: +34 932607787

E-mail address: r.cleries@iconcologia.net (R. Clèries).
}

also associated with cryptorchidism [7] being its etiologic fraction around $10 \%$. Maternal factors during pregnancy as smoking have been also associated although need further investigation $[8,9]$.

An increase in mortality from TC was observed in some Western countries until the beginning of the 1980s followed by a decline afterwards [2]. However, mortality rates from TC in some Eastern and Southern European countries did not decline, with the exception of Italy (4\% decline per annum during the period 1980-1998) [2]. However, trends in TC mortality are difficult to analyze in these studies because of the small number of deaths from this cause.

At present, only two studies analyzed TC mortality trends specifically in Spain, although their period of analysis was comprised between 1950 and the beginning of the 1980s. Both studies showed a slight decrease in mortality at the end of the study period $[10,11]$. The most recent analysis of TC mortality trends in Spain has been carried out during 1994-2003, being reported a non-statistically significant decreasing trend [12].

A time trends study has been carried out through a Bayesian age-period-cohort (APC) analysis, with the aim to estimate the effects of the age and period of death and birth cohort on TC mortality [5-13]. The Bayesian method extracts the necessary information from the data to describe the observed trend. This

1877-7821/\$ - see front matter (c) 2010 Elsevier Ltd. All rights reserved. doi:10.1016/j.canep.2010.03.003 
trend can be projected to the future in the smoothest possible way, achieving sensible predictions in situations when other methods could fail, especially when the number of cases is small, as the case of TC mortality. This Bayesian APC model is particularly interesting because it allows the uncertainty associated with functions of the parameters to be readily explored.

The aims of our study are: (i) to assess recent time trends of TC mortality in Spain based on the most updated data available, which refers to the period 1985-2004, and taking into account the effect of birth cohort, age and period of death, and (ii) to estimate future trends of TC mortality in Spain, period 2005-2019.

In this study Bayesian models have been fitted using WinBUGS [13] through the statistical package $\mathrm{R}[14,15]$. WinBUGS and Rcode for models and graphical representation of the age, period and cohort effects are both described in Appendices A and B.

\section{Materials and methods}

\subsection{Data and population}

The National Institute of Statistics of the Spanish Government (Instituto Nacional de Estadística, INE, http://www.ine.es/) has provided mortality data and population age distribution for the period 1985-2004, as well as future population for the period 20052019. In Spain, throughout the period 1977-1998, the code for TC was 186 according to the ninth revision of the International Classification of Diseases (ICD-9) and since 1998, the code was C62 according to the tenth revision of the ICD (ICD-10). [16]. TC mortality rates were age-standardized using the world standard population. Data was arranged in four 5-year periods (1985-1989, 1990-1994, 1995-1999, 2000-2004), and twelve 5-year age groups (15-20 years to $70-74$ years). These age groups and calendar periods involved 15 (12 age groups +4 periods -1 ) overlapping 10 -year cohorts due to the relation cohort= period-age [17]. The cohort groups were defined by their mid-years starting with 1915 cohort and finishing with the cohort 1985 cohort (1910-1919,..,1945$1954, \ldots, 1980-1989)$, being the central cohort that with central year 1950 (1945-1954). Let us define $A=12, P=4$ and $C=12+4-1=15$ the number of effects for age, period and cohort, respectively.

\subsection{Statistical models}

In this Bayesian analysis, model parameters were assessed through their 95\% credibility intervals (95\% CRI) [18-21]. Let $\theta$ be our model parameter of interest such as a certain age, period or cohort effect being its knowledge formulated as a probability distribution. If an interval has $95 \%$ probability under this distribution, then this interval is called 95\% credibility interval with $95 \%$ credibility. Although it is very common to interpret credibility intervals (CRIs) as confidence intervals, the CRI incorporates information from the prior distribution into the estimate, while confidence intervals are based solely on the data. On the contrary, confidence intervals must be interpreted as a range in which the mean would occur $95 \%$ of the time with repeated sampling, considering the mean to be fixed with no probability distribution [22], whereas CRI interval is the range of values for the parameter of interest and among those, it could include also the median and the mean. In this analysis statistical significance of the parameters was considered when the 95\% CRI did not include the 0 value. The procedure of modeling has been divided into sections described below.

\subsection{Estimation of the annual percent change of rates}

Let $\lambda_{a p}$ be the observed mortality rate (age-specific) for the age group $a$ in the period $p$, where $\lambda_{a p}=Y_{a p} / N_{a p}$, being $Y_{a p}$ and $N_{a p}$ the observed number of deaths and the person-years at risk, respectively, in the age group $a$ and period $p$. Assuming $Y_{a p} \sim \operatorname{Poisson}\left(N_{a p} \lambda_{a p}\right)$ the Bayesian age-drift model used to estimate the annual percent change of increase in the number of cases (EAPCH) [17] during 1985-2004 is

$$
\begin{aligned}
& \log \left(\lambda_{a p}\right)=\eta_{a}+\psi\left(p-p_{0}\right) \\
& \log \left(E\left(Y_{a p}\right)\right)=\log \left(N_{a p}\right)+\eta_{a}+\psi\left(p-p_{0}\right) \\
& \eta_{a} \sim \operatorname{Normal}\left(0, \tau^{2}\right) \\
& \psi \sim \operatorname{Normal}\left(0, \tau^{2}\right) \\
& \tau^{2}=0.001
\end{aligned}
$$

where $\eta_{a}$ the intercept term of the model and $\psi$ the slope of the model. This is a linear model in the natural logarithm scale of the agespecific rates, where $p$ represents the central year of the 5 -year period and $p_{0}$ the central year for the basis period which is 1987 (period 1985-1989). Therefore the EAPCH can be estimated by means of $\left(e^{\psi}-1\right) \times 100$ equation [17]. Non-informative Normal prior distributions [19], with mean 0 and low prior precision (inverse of the variance) $\tau^{2}$, have been assumed for age-drift model parameters.

\subsection{Bayesian age-period-cohort modeling}

Let $H=\left\{\alpha_{1}, \ldots, \alpha_{12}\right\}$ be the set of age effects, $B=\left\{\beta_{1}, \ldots, \beta_{4}\right\}$ be the set of period effects and $\Gamma=\left\{\gamma_{1}, \ldots, \gamma_{15}\right\}$ be the set of cohort effects. The magnitude of the rates can be described by an APC model $\lambda_{a p}=\alpha_{a}+\beta_{p}+\gamma_{c}$, with the birth cohort $c$ derived from period and age such that $c=p-a$ for $c$ in $\{1,2, \ldots, C\}$ [17].

Due to this linear relation between age, period and cohort, the non-identifiability of model parameters problem arise [17] when these parameters have to be represented. We have adapted the Holford approach in order to represent the effects [23-26]. We thus circumvented the non-identifiability problem and presented unique estimates for the period and cohort effects by firstly assuming a cohort slope of zero, implying that period effect influences were entirely responsible for the time trend. A priori evidence suggested that the presentation of the mortality trends should incorporate the well-known benefits from treatment, which should show up as period-related effects. To represent these effects, first we fitted an age-period (AP) model, $\lambda_{a p}=\alpha_{a}+\beta_{p}$ to the observed rates, assuming that the reference period is $1985-1989$. The fitted values obtained through this AP model, $Y^{*}=N_{a p} \times e^{\alpha_{a}+\beta_{p}}$, are introduced into an only-cohort (OC) model as the offset values $Y_{a p}=\log \left(Y_{a p}^{*}\right)+\gamma_{c}$. Note that OC model does not include age effects and must not be considered an age-cohort (AC) model. In the OC model we assumed a certain reference cohort, $\gamma_{R}$, in this analysis the cohort number 8 related with the central birth cohort of 1950 . Then we graphically depicted the age and period effects through the $\left(\alpha_{1}, \ldots, \alpha_{12}, \beta_{1}, \ldots, \beta_{4}\right)$ parameters estimated by means of the AP model, whereas cohort effects, $\left(\gamma_{1}, \ldots, \gamma_{R}=0, \ldots, \gamma_{15}\right)$, were extracted from the $O C$ model [23-26]. By this procedure the standard deviations of the estimated values of the effects can be obtained assuming that the secular trend is related to the period effect. The resulting effects are close to those obtained with the approach of Holford [23-26]. The adaptation of this procedure to the Bayesian framework in R and WinBUGS (see Appendix A) could lead to slightly higher variability than that of the frequentist one due to the simulation method internally implemented in WinBUGS [13].

\subsection{Prior distributions for age, period and cohort effects and their prior adaptive precision parameter}

As TC is a mortality cause with low number of cases, trends could be modeled through specific smoothing of model para- 
meters. A second order random walk [19] (RW2) has been assumed for all effects, whereas second order differences of this RW2 have been constrained for age parameters, assuming that one second order difference is estimated as the mean value on the previous and subsequent second order differences $[18,20]$. Thus, the age effect has been constrained to

$$
\begin{aligned}
& \alpha_{i} \mid \alpha_{j}, j \neq i \sim N\left(\mu_{\alpha_{i} \mid \alpha_{j}, j \neq i}, \tau_{\text {age }}\right) \\
& \mu_{\alpha_{1} \mid \alpha_{2}, \alpha_{3}}=2 \alpha_{2}-\alpha_{3} \\
& \mu_{\alpha_{2} \mid \alpha_{1}, \alpha_{3}, \alpha_{4}}=\frac{2 \alpha_{1}+4 \alpha_{3}-\alpha_{4}}{5} \\
& \mu_{\alpha_{i} \mid \alpha_{i+1}, \alpha_{i+2}, \alpha_{i-1}, \alpha_{i-2}}=\frac{4 \alpha_{i-1}+4 \alpha_{i+1}-\alpha_{i-2}-\alpha_{i+2}}{6}, 3 \leq i \leq A-2 \\
& \mu_{\alpha_{A-1} \mid \alpha_{A}, \alpha_{A-2}, \alpha_{A-3}}=\frac{2 \alpha_{A}+4 \alpha_{A-2}-\alpha_{A-3}}{5} \\
& \mu_{\alpha_{A} \mid \alpha_{A-1}, \alpha_{A-2}}=2 \alpha_{A-1}-\alpha_{A-2} \\
& \tau_{\text {age }}=K_{s} \frac{1}{\sigma_{\text {age }}^{2}} \\
& \sigma_{\text {age }} \sim \text { Uniform }(0.01,1)
\end{aligned}
$$

being $\alpha_{i}$ the effect of the $i$ th age group, $(1, \ldots, A=12), \mu_{\alpha_{i} \mid \alpha_{j}, j \neq i}$ its prior mean value, $\sigma_{\text {age }}$ its prior standard deviation and $\tau_{\text {age }}$ its prior precision (inverse of the prior variance) [19]. Prior standard deviation of the parameters have been modeled through uniform prior distributions on interval $[0.01,1]$ which they are expected to perform well unless the number of levels of the variable is approximately below five [21]. Note that precision includes an extra parameter denoted as $K_{s}$ which allows for adapting prior precision of these models to different diseases. This adaptive parameter has been assumed to be the same for all effects. Period parameters, $\left\{\beta_{1}, \ldots, \beta_{4}\right\}$, have been modeled through second degree autoregressive smoothing as

$$
\begin{aligned}
& \beta_{1}=0 \\
& \beta_{2} \sim N\left(0, \tau_{\text {period }}\right) \\
& \beta_{i} \mid \beta_{i-1}, \beta_{i-2} \sim N\left(\mu_{\beta_{i} \mid \beta_{i-1}, \beta_{i-2}}, 10^{-6} \tau_{\text {period }}\right), 3 \leq i \leq P \\
& \mu_{\beta_{i} \mid \beta_{i-1}, \beta_{i-2}}=2 \beta_{i-1}-\beta_{i-2}, 3 \leq i \leq P \\
& \tau_{\text {period }}=K_{s} \frac{1}{\sigma_{\text {period }}^{2}} \\
& \sigma_{\text {period }} \sim \text { Uniform }(0.01,1)
\end{aligned}
$$

Note that $\beta_{1}=0$ refers to the reference period, in this case the first period. In the same line, cohort parameters, $\left\{\gamma_{1}, \ldots, \gamma_{R}=\right.$ $\left.0, \ldots, \gamma_{15}\right\}$ were modeled through

$$
\begin{aligned}
& \gamma_{1} \sim N\left(0, \tau_{\text {cohort }}\right) \\
& \gamma_{2} \sim N\left(0, \tau_{\text {cohort }}\right) \\
& \gamma_{i} \mid \gamma_{i-1}, \gamma_{i-2} \sim N\left(\mu_{\gamma_{i} \mid \gamma_{i-1}, \gamma_{i-2}}, \tau_{\text {cohort }}\right), 3 \leq i \leq R-1 \\
& \gamma_{R}=0 \\
& \gamma_{i} \mid \gamma_{i-1}, \gamma_{i-2} \sim N\left(\mu_{\gamma_{i} \mid \gamma_{i-1}, \gamma_{i-2}}, \tau_{\text {cohort }}\right), R+1 \leq i \leq C, \\
& \mu_{\gamma_{i} \mid \gamma_{i-1}, \gamma_{i-2}}=2 \gamma_{i-1}-\gamma_{i-2} \\
& \tau_{\text {cohort }}=K_{s} \frac{1}{\sigma_{\text {cohort }}^{2}} \\
& \sigma_{\text {cohort }} \sim \text { Uni form }(0.01,1)
\end{aligned}
$$

being $R=8$ the central and reference cohort considered in our study.

\subsection{Selecting adaptive precision}

Deviance Information Criterion (DIC) has been used as Bayesian model selection criterion [22]. DIC is the sum of the posterior deviance and the effective number of model parameters $(\mathrm{pD})$. The lowest value of DIC indicates the best fitting model with best outof-sample predictive power [22] and, therefore the selected one to perform predictions. We tested DIC values for AP, AC and APC models in five scenarios, depending on the value for $K_{s}$. For this analysis we have evaluated $K_{s}$ in five different situations, such that $K_{s} \in\{1,0.1,0.01,0.001,0.0001\}$. We have denoted this parameter as the adaptive precision parameter. The adaptive precision parameter selected has been used (1) in the AP and AC models for graphic representation of rates as well as (2) for the APC model used for projections.

\subsection{Modeling projections}

An APC model with RW2 for all parameters using formulation described in Eqs. (2-4) have been fitted to 1985-2004 data. The RW on the period and cohort model parameters allows extrapolating future values for these parameters and, therefore, the future trends of the rates, whereas the RW on age effects allows exponential smoothing of the rates [18-20]. In this line and due to the relation cohort $=$ period-age, the future number of deceased TC cases has been predicted for 3 periods (2005-2009, 2010-2014 and 2015-2019) and, therefore, for 2 future cohorts (1985-1995, 1990-1999 and 1995-2004). The expected number of TC deceased cases for 2005-2019 has been obtained through the Bayesian APC model selected taking into account the value of the adaptive parameter, $K_{s}$. The age-drift model of Eq. (1) has been also used to estimate future EAPCH during period 2005-2019 once predictions have been performed, using as $p_{0}$ the year 2007 which is the central one for period 2005-2009.

\section{Results}

During 1985-2004, a total of 763 deaths (38 deaths per year) from TC were observed in Spain. From these, 581 deceased cases (29 deaths per year) were found in the age groups 15-54 years old.

Table 1 shows the procedure for the selection of the adaptive precision parameter $K_{s}$. The influence of the adaptive precision parameters can be assessed through the different DIC values. In this analysis we have selected the value 0.001 because AP, AC and APC models tested showed the lowest DIC values.

Fig. 1 depicts the age, period and cohort effects during the period 1985-2004 and their 95\% CRI. The age effect showed an exponential rising before the age of 30 , climbing to a crude TC mortality rate near 5 cases per 1,000,000 men-years. After this age, there was a dramatic decrease, reaching a stabilization of rates near the age of 40 . Curvature of the cohort effect showed a decrease on risk from the 1935 birth cohort and a stabilization near to the 1965 birth cohort with small rise for the youngest cohorts. The period effect witnessed a continuous decrease after the initial period of death (1985-1989, reference period).

The age-drift model enabled to estimate the EAPCH during 1985-2004. The EAPCH was $-2.41 \%$ (95\% CRI: $-3.65 \%$; $-1.13 \%)$, whereas TC mortality rates decreased by $-1.18 \%$ (95\% CRI: $-2.60 \%$; $-0.31 \%$ ) when the analysis was restricted to age groups $15-54$. However, EAPCH for men younger than 35 was not found to be statistically significant (EAPCH $-0.97 \%$; $95 \%$ CRI: $-2.78 \%$; $+0.86 \%$ ).

Fig. 2 shows age-specific projections for TC mortality rates in Spain during 2005-2019 and their 95\% CRI. Based on these projections, a leveling off for TC mortality rates among Spanish population would be reached among men older than 35 , whereas higher variability was observed among men younger than 35 . It 
Table 1

Procedure for the selection of the adaptive precision parameter using Deviance Information Criterion (DIC) and the effective number of model parameters (pD).

\begin{tabular}{|c|c|c|c|c|c|c|c|c|c|c|}
\hline \multirow{2}{*}{$\begin{array}{l}K_{s}: \text { adaptive precision parameter } \\
\text { Model }\end{array}$} & \multicolumn{2}{|l|}{$K_{s}=1$} & \multicolumn{2}{|l|}{$K_{s}=0.1$} & \multicolumn{2}{|c|}{$K_{s}=0.01$} & \multicolumn{2}{|c|}{$K_{s}=0.001^{\mathrm{a}}$} & \multicolumn{2}{|c|}{$K_{s}=0.0001$} \\
\hline & DIC & $\mathrm{pD}$ & DIC & $\mathrm{pD}$ & DIC & $\mathrm{pD}$ & DIC & $\mathrm{pD}$ & DIC & $\mathrm{pD}$ \\
\hline Age-period & 309.28 & 12.19 & 307.13 & 12.26 & 306.18 & 11.16 & 299.15 & 14.25 & 299.18 & 14.27 \\
\hline Age-period-cohort & 250.21 & 24.12 & 243.98 & 24.16 & 241.98 & 25.01 & 236.45 & 25.35 & 239.41 & 25.52 \\
\hline
\end{tabular}

DIC: Deviance Information Criterion. DIC = PDev + pD. PDev: posterior deviance; pD: Effective number of model parameters.

a The adaptive precision parameter selected due to DIC value is the minimum observed among models. Note the influence of the precision parameters on the predictive performance of the models due to model with lowest DIC value best predictive performance shows.

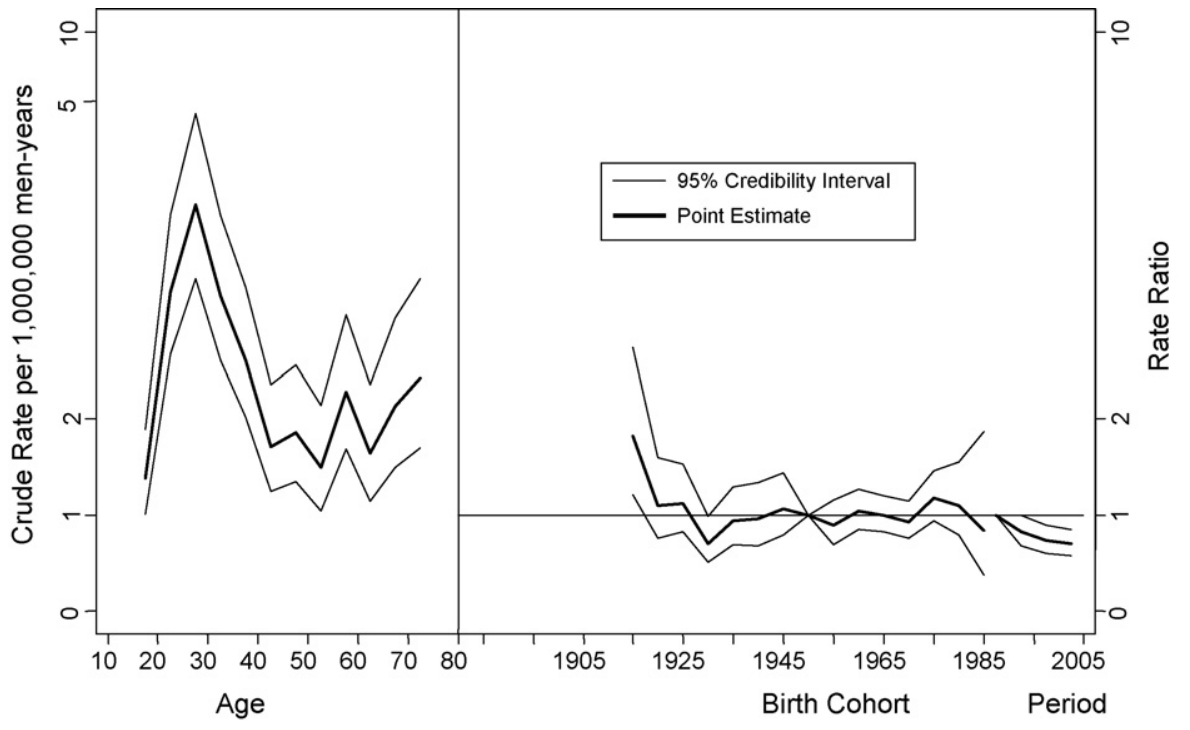

Fig. 1. Age, period and cohort effects for testicular cancer mortality rates in Spain during the period 1985-2004.
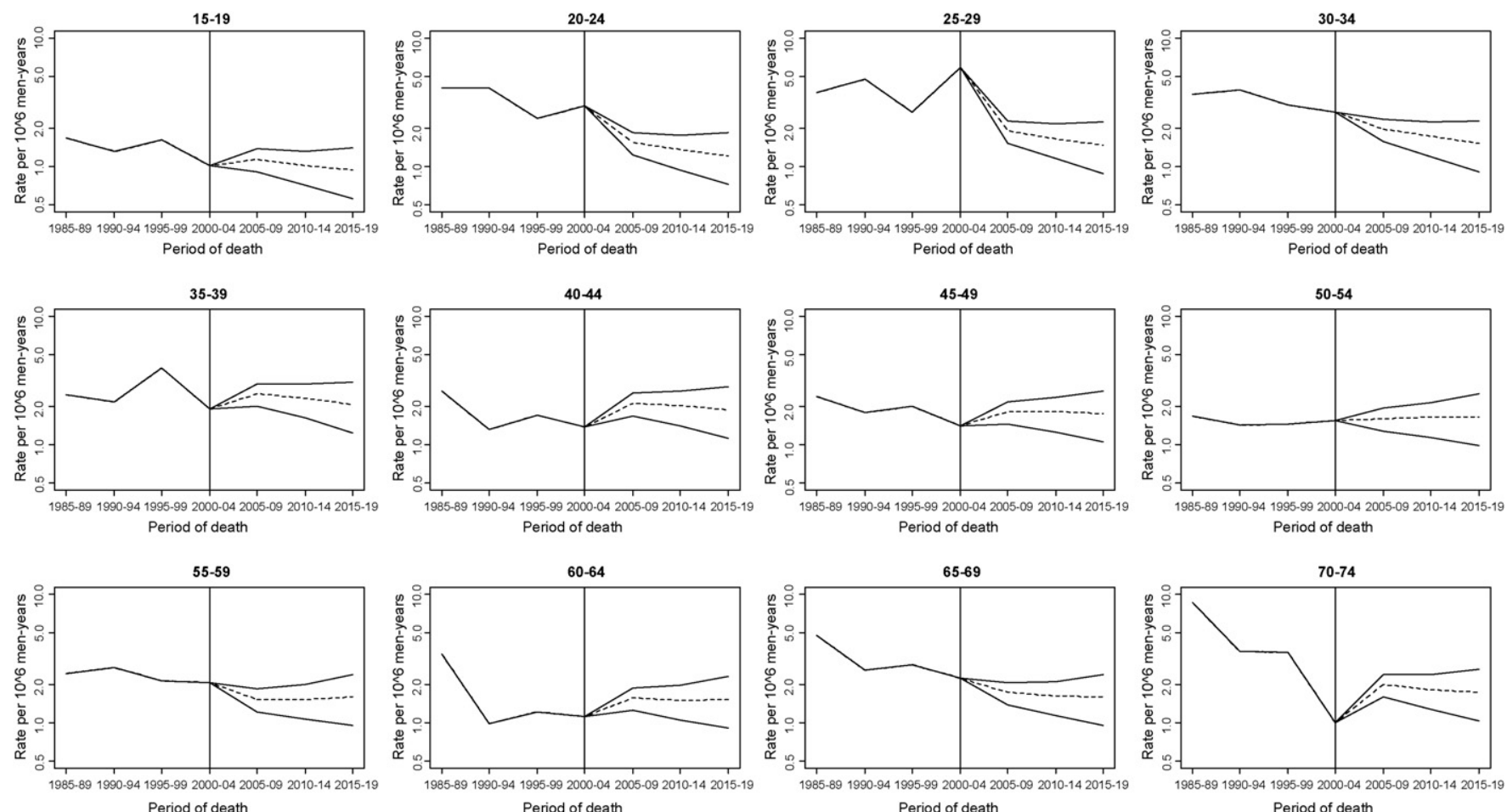

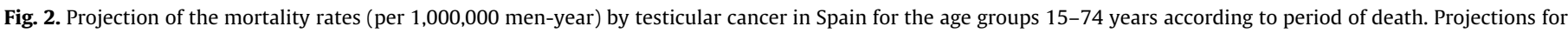
periods 2005-2009, 2010-2014, 2015-2019 (dashed line - - -) and their 95\% credibility intervals (solid line -). 
Table 2

Expected number of deaths from testicular cancer in Spain for periods 2005-2009, 2010-2014, 2015-2019: age groups 15-34, 15-54, 55-74 and 15-74.

\begin{tabular}{|c|c|c|c|c|}
\hline Period & $\begin{array}{l}15-34 \\
(N)\end{array}$ & $\begin{array}{l}35-54 \\
(N)\end{array}$ & $\begin{array}{l}55-74 \\
(N)\end{array}$ & $\begin{array}{l}15-74 \\
(N)\end{array}$ \\
\hline 1985-1989 & 90 & 60 & 69 & 219 \\
\hline 1990-1994 & 112 & 40 & 40 & 192 \\
\hline 1995-1999 & 78 & 60 & 42 & 180 \\
\hline 2000-2004 & 99 & 43 & 30 & 172 \\
\hline Projections-period & ( $N$ and $95 \%$ CRI) & ( $N$ and $95 \%$ CRI) & ( $N$ and $95 \% \mathrm{CRI}$ ) & ( $N$ and $95 \% \mathrm{CRI}$ ) \\
\hline 2005-2009 & $66(43 ; 83)$ & $63(46 ; 84)$ & $37(29 ; 43)$ & $166(128 ; 218)$ \\
\hline 2010-2014 & $58(47 ; 91)$ & $66(43 ; 88)$ & $39(26 ; 48)$ & $159(102 ; 249)$ \\
\hline 2015-2019 & $51(29 ; 90)$ & $62(42 ; 99)$ & $28(18 ; 57)$ & $145(75 ; 283)$ \\
\hline EAPCH 2005-2019 (95\% CRI) & $-2.3(-4.27 ;-0.31)^{\mathrm{a}}$ & $-0.71(-1.42 ;+0.18)$ & $-0.52(-1.05 ;+0.12)$ & $-1.12(-1.54 ;-0.63)^{\mathrm{a}}$ \\
\hline
\end{tabular}

$N$ : number of expected death cases by testicular cancer in Spain (posterior median); 95\% CRI: 95\% credibility interval.

EAPCH 2005-2019: estimated annual percent change of TC mortality rates during period 2005-2019.

a $95 \%$ CRI does not include 0 value.

should be noted that, based on our simulations, the increase in ten cases in some age groups could affect these trends. According to these results, Table 2 describes projections for the TC mortality in Spain on the basis of mortality data from the period 1990-2004 and for age groups 15-34, 35-54 and 55-74. While the mean number of TC cases decreases over time, 95\% CRI are overlapped along periods showing TC mortality leveling off. Future mortality rates were estimated through the expected number of deaths from TC during 2005-2019. A decreasing trend in TC mortality rates for 2005-2019 was estimated (EAPCH -1.12\%; 95\% CRI: $-1.54 \%$; $-0.63 \%$ ) mainly attributed to the significant decreasing trend of TC mortality rates among men younger than 35 years $(\mathrm{EAPCH}-2.3 \%$; 95\% CRI: $-4.27 \%$; $-0.31 \%)$.

\section{Discussion}

Our study systematically assesses the effect of age, period and birth cohort on TC in Spain showing the importance of the period influence when all age groups were included in the analysis. A decreasing mortality trend of $-2.41 \%$ (95\% CRI: $-3.65 \%$; $-1.13 \%$ ) per annum was observed during 1985-2004, whereas decreasing mortality trend for the age groups $15-54$ was smaller. We have shown a modeling framework for projections of TC mortality when the number of cases is small. On the other hand, we presented a Bayesian modeling approach to age-period-cohort analysis in order to deal with those locations with low number of cases and, therefore, small rates which could lead to computational problems when projections are performed $[18,20]$.

Since the mid 1970s, TC mortality has declined in several European countries due to advances in treatment [27], and this has translated to cohorts of men with successively lower risk of death from this disease [2]. A recent study of incidence and mortality from TC in 22 European countries showed that the first beneficiaries of therapy in these populations were those men born after the 1970s [27]. Period-specific trends shown a reduction in risk of death in Spain through periods 1953-1977 [11] and 1951-1983 [10]. Both studies showed an increase of mortality from TC among young and middle-aged males and a decline in the older age groups. Our study results show a decreasing trend is expected for men younger than 35 and a leveling off for those older than 35 for period 1985-2019. The number of TC deceased cases decreased about 26\% between 1985 and 1994. Mortality risk from TC reaches its peak between ages 20 and 34 and drops to a stabilization of rates near the age of 40 (age effect), as it has been observed in previous studies in Western and Eastern European countries [2]. The increased incidence risk of testicular cancer was detected for men 15-44 in most industrialized countries [2,28-32]. Spain has noted recently the most rapid inclines in TC incidence among European countries, although it has one of the lowest TC incidence and mortality rates in Europe [1,2].

The decrease of $-2.41 \%$ per annum in TC mortality rates through the period of study in age groups 15-74 could be attributable to the introduction of cisplatin as a therapeutic agent for advanced germ-cell tumors [27] and best-practice tumor management $[33,34]$ (period effects). Down-staging due to testicular self-examination and screening are effective methods for an early detection of this cancer, and the introduction of various systemic adjuvant therapies have an effect on TC survival [35,36]. A recent study on TC survival showed little differences between western European registries which were mainly attributable to differing stage at diagnosis [37]. The success of (1) cisplatincontaining chemotherapy - which has been proven to be the most effective treatment for non-seminoma testicular cancer - in terms of reduction in mortality and (2) an adequate patient management are also responsible for favorable trends [12,38]. These are reflected on improvements in relative survival rates (RSR) among European TC patients diagnosed through the 1990s (5-year RSR Europe: $93 \%$ in $1990-1994$ vs. $93.8 \%$ in 1995-1998, 5-year RSR Spain: $89.3 \%$ in $1990-1994$ vs. $96.7 \%$ in $1995-1999$ ) [39,40], being 10\% higher than TC 5-year RSR during the period 1983-1985 [41].

In the graphical representation of the effects we have $a$ priori assumed that regular trend for TC mortality was a result of period influences, as previously described in other studies [2]. If this hypothesis was erroneous, a bias in all effects may be incurred [17]. In agreement with our hypothesis, this fact shows the predominant period effect. The same conclusion could be extracted from the sub-analysis for the age groups 15-54, although the decrease of mortality was smaller. In this line, the analysis of Spanish AgeStandardized TC mortality rates since 1970 shows a decreasing trend for all age groups (data not shown and extracted from http:// www-dep.iarc.fr/: EAPC for $15-74=-2.65 \%$ with $95 \%$ CRI: $-3.25 \%$; $-1.23 \%$; EAPC for $15-54=-1.61 \%$ with $95 \%$ CRI: $-2.51 \% ;-0.71 \%$ ).

Projections by model converged to stabilization of the decreasing trend of TC mortality rates with the exception of age groups 15-34 years old. Several limitations should be mentioned in relation with projections. First, projected trends were based on small numbers of TC cases and interpretation at this particular level - trends by age group - could be difficult. Importantly, we have evaluated that the increase or decrease of ten or more cases in each age group could dramatically modify the projected trends. Second, Spanish population growth could also modify these trends, with special mention to immigration effect [42]. The annual increase of immigrants in Spain could lead to a slight rise in the number of cases. However, the known effect of ageing in projections [43] should not modify these results due to TC is more frequent among 15-34 years old. Despite the increase in 
incidence, the stabilization in TC mortality could be also noted due to high RSR observed among Spanish population [39].

Although the median number of total projected cases for all age groups decreased through period 2005-2019, 95\% CRI were overlapped in all age groups. These last results lead to conclude that no longer decreasing trend of TC mortality is expected in Spain in these age groups, being this finding translated into a TC mortality leveling off in the years to come.

Our modeling framework allowed estimation of the future TC mortality trends, projecting them into the future in the smoothest possible way, achieving sensible predictions in a framework of modeling when other methods could fail when the number of cases is small. We have defined a predictive precision parameter, $K_{s}$ to take into account in projections. Even predictive precision parameter has been considered as a constant of order $10^{-6}$ in Bayesian age-period-cohort modeling $[18,20]$, its impact has not been assessed in previous Bayesian APC analysis nor in the predictions of the burden of a cancer site with low mortality rates as TC. We ascertained $K_{s}$ performance through different scenarios assessing the DIC impact, the criteria for selecting the APC model which was a key part in our analysis. Note that $K_{s}$ affects posterior precision of model parameters and, therefore, projected number of cases in a future time period. We recommend sensibility analysis for this parameter which will allow adapting our models to other diseases.

In conclusion, this study provides a compromise between statistical analysis and description of mortality trends from TC in Spain, where management of TC and advances in therapy led to the decrease of mortality from this disease during the period 1985-
2004. The stabilization of the falls in rates over the next 15 -year period could confirm that mortality from TC should reach a plateau as it has been observed in some western countries [2,12]. Even if prognosis is considered as good, a group with bad prognosis could still remain and diagnostic delay, defined as the time elapsing from the onset of tumor symptoms to the day of diagnosis, is a way to evaluate the rapidity of diagnosis [44]. Thus, despite the recent favorable trend due to adequate treatment and improvements in curability from this disease in the last decade [34,35] the recently detected rise in incidence from TC in Spain should keep a special surveillance for this disease in the years to come among Spanish men.

\section{Conflict of interest}

Authors declare that there is no conflict of interest with any financial organization regarding the material discussed in the manuscript.

\section{Acknowledgements}

Partial support has been received from the Instituto de Salud Carlos III of the Spanish Government (C03/09, C03/10) and from the Agencia de Gestio d'Ajuts Universitaris i de Recerca of the Catalan Government (Grants 2003XT 00023 and 2006 BE00614). Authors want to acknowledge anonymous reviewer's comments who improved substantially the exposition on the statistical methods section. 


\section{Appendix A. $R$ and WinBUGS codes}

\section{A.1. Graphical representation}

The WinBUGS code for the analysis is described in the following two models

\section{A.1.1. Model AP}

\#MODEL AGE-PERIOD

model

\{

\#\#\# Fit of the Period and age

for (n in 1:N) \{

Y.ap [n] dpois (expected.Y.ap [n]);

\# Modeling rate

$\log (\operatorname{expected.Y.ap[n])~<-~} \log (\mathrm{N} \cdot \mathrm{ap}[\mathrm{n}])+$ alpha [age[n] ] + beta [period [n] ] \} \#\#\#\#\#\#\#\#\#\#\#\#\#\#\#\#\#\#\#\#\#\#\#\#\#\#\#\#\#\#\#\#\# PERIOD: REFERENCE FIRST PERIOD 1985-89 betamean $[1]<-0.0$;

betaprec [1] <- tau2.period;

betamean $[2]<-0.0$;

betaprec [2] <- tau2.period:

for $(j$ in $3: J)\{$

betamean $[j]<-2 * \operatorname{beta}[j-1]$ - beta $[j-2]$;

betaprec $[j]<-$ tau2.period; \}

beta $[1]<-0$

for $(j$ in $2: J)$.

beta $[j] \sim$ dnorm (betamean $[j]$, betaprec $[j])$; \}

\#\#\#\#\#\#\#\#\#\#\#\#\#\#\#\#\#\#\#\#\#\#\#\#\#\#\#\#\#\#\#\#\#\#\#\#\#\#\#\#PERIOD: prior standard deviation tau2.period<-K.s*pow (sigma.period, -2)

sigma.period dunif $(0.01,1)$;

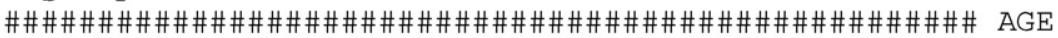

alphamean[1] <- 2*alpha[2] - alpha[3];

alphamean [2] <- (2*alpha[1] + 4*alpha[3] - alpha[4])/5;

for (i in $3:(I-2))\{$

alphamean[i] <- (4*alpha[i-1] + 4*alpha[i+1]- alpha[i-2]

$\bar{j}$

alphamean [I-1] <- (2*alpha[I] + 4*alpha[I-2] - alpha[I-3])/5;

alphamean $[I]<-2 * a l p h a[I-1]$ - alpha[I-2];

for ( $i$ in $1: I)\{$

alphaprec [i] <- tau2.age;

\}

for $(i$ in $1: I)\{$

alpha[i] dnorm(alphamean[i], alphaprec [i]);

\}

\#\#\#\#\#\#\#\#\#\#\#\#\#\#\#\#\#\#\#\#\#\#\#\#\#\#\#\#\#\#\#\#\#\#\#\#\#\#\#\#\#GE: prior standard deviation tau2.age<-K.s*pow (sigma.age, -2);

sigma.age $\sim$ dunif $(0.01,1)$;

\} 
A.2. Model with COHORT (only-cohort model, OC model):

\# OC MODEL: Model with only COHORT EFFECT and offset the fitted values from an AP model

model

\{

\#\#\# Fit of the Cohort effect

for $(\mathrm{n}$ in $1: \mathrm{N}) \quad\{$

Y.ap [n] dpois (expected.Y.ap[n]);

\# Modeling rate

$\log ($ expected.Y.ap $[n])<-\log ($ offset $[n])+$ gamma [cohort $[n]]$

\}

\#\#\# COHORT : prior standard deviation

tau2. cohort $<-0.001$ *pow (sigma. cohort, -2$)$

sigma.cohort dunif $(0.01,1)$;

\#\#\# COHORT REF 1950: 8 cohort

gammamean $[1]<-0.0$;

gammaprec [1] <- tau2. cohort

gammamean [2] $<-0.0$;

gammaprec [2] <- tau2. cohort

for $(\mathrm{k}$ in $3: \mathrm{K})$ \{

\#gammamean $[\mathrm{k}]<-2$ <gamma [k-1] - gamma [k-2];

gammamean $[\mathrm{k}]<-0$

gammaprec $[\mathrm{k}]<-$ tau2. cohort;

\}

for $(\mathrm{k}$ in $1: 7)\{$

gamma [k] dnorm (gammamean [k], gammaprec [k]) ;

\}

gamma $[8]<-0$

for $(\mathrm{k}$ in $9: \mathrm{K})\{$

gamma [k] d dnorm (gammamean [k], gammaprec [k]);

\}

Please cite this article in press as: Clèries R, et al. Monitoring the decreasing trend of testicular cancer mortality in Spain during 20052019 through a Bayesian approach. Cancer Epidemiology (2010), doi:10.1016/j.canep.2010.03.003 
A.3. $R$-code to run the models through library R2WinBUGS is

\#\#\# Script Graphics in R using Sequential Approach: Assume Time trend on Period effect and no slope for Cohort effects

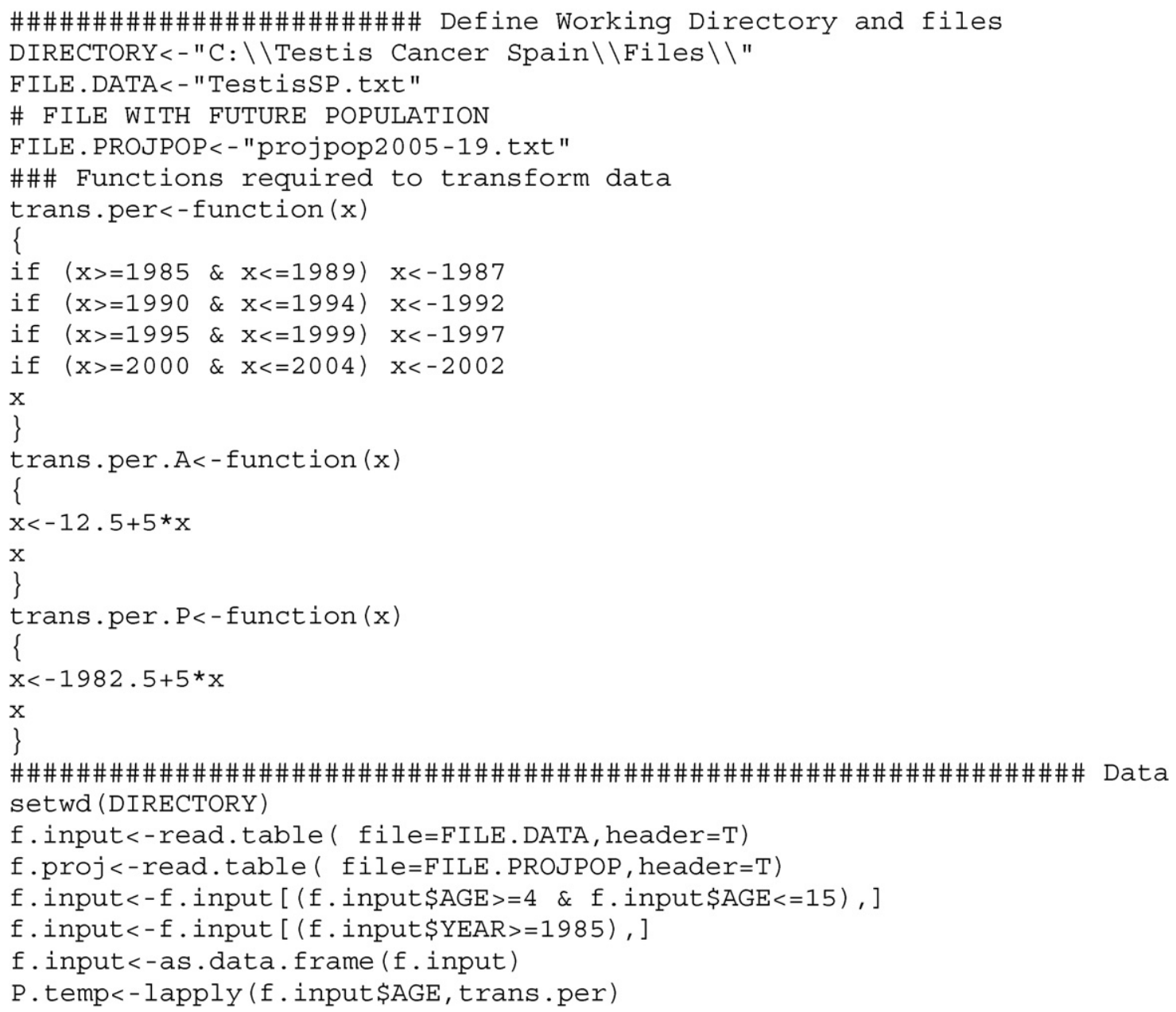


P. temp<-as. numeric (P. temp)

ED . temp $<-$ f . input $\$ A G E * 5-3+0.5$

P. temp<-as.numeric (lapply (f. input\$YEAR, trans.per) ) +0.5

D. temp $<-\mathrm{f}$. input\$DEATHS

Y. temp $<-f$. input $\$ P O B$

xtest $<-$ cbind (ED. temp, P. temp, D. temp, Y . temp)

xtest<-as.data.frame (xtest)

names (xtest) <-c ("A", "P", "D", "Y")

xtest<-aggregate (xtest [, 3:4], list (xtest\$A, xtest\$P), sum)

names (xtest) <-c ("A", "P", "D", "Y")

xtest $\$ C<-$ xtest\$P - xtest\$A

\#\#\#\#\#\#\#\#\#\#\#\#\#\#\#\#\#\#\#\#\#\#\#\#\#\#\#\#\#\#\#\#\#\#\# Prepare dataset

xtest. $1<-x$ test

Period<-c (as.numeric (factor (xtest.1\$P)))

Age $<-c($ as.numeric (factor (xtest.1\$A)))

Cohort<-c (as.numeric (factor (xtest.1\$C)) )

Population<-c (xtest. $1 \$ Y)$

Deaths $<-x t e s t .1 \$ D$

\#\#\#\#\#\#\#\#\#\#\#\#\#\#\#\#\#\#\#\#\#\#\#\#\#\#\#\#\#\#\#\# WINBUGS PARAMETERS

\# N.A: Number of Age groups

\# N.P: Number of Periods

\# N.C: Number of Cohorts

N. A $<-12$

N. P $<-4$

N. $\mathrm{C}<-\mathrm{N} \cdot \mathrm{A}+\mathrm{N} \cdot \mathrm{P}-1$

library (R2WinBUGS)

parameters<-c ("alpha", "beta", "expected.Y.ap")

\#\#\#\#\#\#\#\#\#\#\#\# Pre-Define Initials for each chain: 3 Chains

inits $1<-$

list ( sigma.age=1, sigma.period=1, alpha =c (rnorm (N.A, 0, 0.1)), beta=c (NA, rn

$\operatorname{orm}(\mathrm{N} . \mathrm{P}-1,0,0.1)))$

inits $2<-$

list ( $\operatorname{sigma}$. age=1, sigma.period=1, alpha $=\mathrm{C}(\operatorname{rnorm}(\mathrm{N} . \mathrm{A}, 0,0.1))$, beta $=\mathrm{C}(\mathrm{NA}, \mathrm{rn}$ $\operatorname{orm}(\mathrm{N} . \mathrm{P}-1,0,0.1)))$

inits $3<-$

list ( sigma.age=1, sigma.period=1, alpha =c (rnorm (N.A, 0, 0.1)), beta=c (NA, rn $\operatorname{orm}(\mathrm{N} . \mathrm{P}-1,0,0.1)))$

dadesl<-list $(\mathrm{N}=$

$N . A * N . P$, age $=$ Age, period=Period, $Y . a p=$ Deaths, $N$. ap=Population, I=N.A,

$\mathrm{J}=\mathrm{N} . \mathrm{P}, \mathrm{K} . \mathrm{S}=0.001$ )

\#\#\#\#\#\#\#\#\#\#\#\#\#\#\#\#\#\# Define Initials

initials<-list (inits1, inits2, inits3)

\#\#\#\#\#\#\#\#\#\#\#\#\#\#\#\#\#\#\#\#\#\#\#\#\#\#\#\# Run WinBugs

itera $<-5000$

burnin $<-2000$

thins $<-1$

dataset $1<-$ dades 1

\#\# Step 1: AP with AR in A+P: Use code A.1

exec.Bugs.APC.HOLF1 <- bugs (dataset1, initials, parameters, "MODAP-

R.txt", n.chains=3, n.iter=itera,

n.burnin=burnin, n.thin=thins, bugs.directory="c:/Program

Files/WinBUGS14/", debug=T)

\#\#\# Extract the fitted values

mu. offset<-

as. numeric (exec. Bugs. APC.HOLF1\$summary $[(N \cdot A+N \cdot P):((N \cdot A+N \cdot P)+(N \cdot A * N \cdot P-$

1) ), 1])

\#\# Model Cohort effects with fitted values from the AP model as offset

\# Note: tauc refers to tau.cohort on the set of equations (3) 
\# Note we define cohort 1950, cohort number 8 as reference one, so we assume gamma[8] to be NA in the initials

inits $1<-1$ ist $(\operatorname{sigma} . \operatorname{cohort}=1, \operatorname{gamma}=\mathrm{C}(\operatorname{rnorm}(7,0,0.1), \operatorname{NA}, \operatorname{rnorm}(7,0,0.1)))$

inits $2<-1$ ist ( $\operatorname{sigma}$. cohort $=1, \operatorname{gamma}=\mathrm{C}(\operatorname{rnorm}(7,0,0.1), \mathrm{NA}, \operatorname{rnorm}(7,0,0.1)))$

inits $3<-1$ ist $(\operatorname{sigma}$. cohort $=1, \operatorname{gamma}=\mathrm{C}(\operatorname{rnorm}(7,0,0.1), \operatorname{NA}, \operatorname{rnorm}(7,0,0.1)))$

parameters<-c ("gamma")

dades $2<-$ Iist $(\mathrm{N}=48$, cohort $=$ Cohort, $Y$. ap=Deaths, offset $=$ mu. offset, $\mathrm{K}=$

N.C)

\#\#\#\#\#\#\#\#\#\#\#\#\#\#\#\#\#\#\#\#\#\#\#\#\#\#\#\#\#\# Define Initials

initials<-list (inits1, inits2, inits3)

\#\#4. Run WinBugs

itera $<-5000$

burnin<-2000

thins $<-1$

dataset $2<$-dades 2

\#\#\#\#\#\#\#\# Only-Cohort Model: Model with only cohort effect, Model A.2 exec.Bugs.APC.HOLF2 <- bugs (dataset2, initials, parameters, "MODOC-

R.txt", n.chains $=3$, n.iter=itera,

n.burnin=burnin, n.thin=thins, bugs.directory="c:/Archivos de

Programa/WinBUGS14/", debug $=\mathrm{T}$ )

\#\#\#\#\#\#\#\#\#\#\#\#\#\#\#\#\# Generate Graphics

a.pt <- seq $(17.5,72.5,5)$

p.pt <- seq $(1987.5,2002.5,5)$

c.pt $<-\operatorname{seq}(1915,1985,5)$

a.meff<-exec.Bugs.APC. HOLF1\$summary $[1: 12, \mathrm{c}(5,3,7)]$

p. $\operatorname{meff} 1<-\mathrm{C}(0,0,0)$

p.meff $2<-$ exec.Bugs.APC. HOLF 1 \$summary $[13: 15, c(5,3,7)]$

p.meff<-rbind (p.meff $1, p . \operatorname{mef} f 2$ )

C. meff $1<-$ exec.Bugs.APC. HOLF 2 \$summary $[1: 7, \mathrm{C}(5,3,7)]$

c. $\operatorname{meff} 2<-\mathrm{C}(0,0,0)$

C. meff $3<-$ exec.Bugs.APC. HOLF 2 \$summary $[8: 14, \mathrm{C}(5,3,7)]$

$c \cdot \operatorname{mef} f<-r b i n d(c \cdot \operatorname{meff} 1, c \cdot \operatorname{meff} 2, \mathrm{c} \cdot \operatorname{mef} f 3)$

$\mathrm{x} 11()$

$\operatorname{par}(\operatorname{mfrow}=\mathrm{C}(2,1), \operatorname{mar}=\mathrm{C}(3,3,0.2,3), \operatorname{mgp}=\mathrm{C}(3,1,0) / 1.8)$

matplot( $\mathrm{NA}$,

$x l a b=" 1, x l i m=c(15,200), \quad x a x t=" n "$, yaxt="n", $y l a b=" ", y l i m=c(0,10))$

matlines ( a.pt, exp(a.meff) * 1000000, type="1", $1 \mathrm{wd}=\mathrm{c}(3,1,1), \quad 1 \mathrm{ty}=1, \mathrm{col}=1)$

matlines ( c.pt-1800, $\exp$ ( c.meff),

$l w d=c(3,1,1), \quad l t y=1, c o l=1)$

matlines ( p.pt-1800, exp (p.meff),

$1 \mathrm{wd}=\mathrm{c}(3,1,1), \quad 1 \mathrm{ty}=1, \mathrm{col}=1)$

ytck <- as.vector ( outer $\left(\mathrm{c}(0,1,2,6), 10^{\wedge}(-2: 1), " * \|\right)$ )

axis ( $\operatorname{side}=1, \operatorname{at}=\operatorname{seq}(0,80,10)$ )

axis ( $\operatorname{side}=1, a t=\operatorname{seq}(0,80,10), 1$ abels $=N A)$

axis ( $\operatorname{side}=1, a t=\operatorname{seq}(1905,2015,20)-1800,1$ abels $=\operatorname{seq}(1905,2015,20))$

$\operatorname{axis}(\mathrm{side}=1, \operatorname{at}=\mathrm{seq}(1905,2015,10)-1820$, labels $=\mathrm{NA})$

axis ( $\mathrm{side}=1, a t=35, \mathrm{label} s=" \mathrm{Age} ", \mathrm{line}=1, \mathrm{tcl}=0$ )

axis ( side=1, at $=1955-1800$, labels="Birth Cohort

Period", line $=1, \operatorname{tcl}=0$ )

axis ( side=2, at=ytck, labels=paste ( ytck ) )

mtext ( side=2, "Crude Rate per 1,000,000 men-years", line=2)

axis ( $\operatorname{side}=4$, at=ytck, labels=paste ( ytck ) )

mtext ( $\mathrm{side}=4$, "Rate Ratio", line=2 )

segments (1890-1810, 1, 2025-1820, 1)

abline $(\mathrm{v}=80)$

abline $(\mathrm{V}=1980)$

\# END GRAPHIC REPRESENTATION 


\section{Appendix B. Model for projections}

This model uses RW2 for all parameters defined through Eqs. (2-4) adding a constraint for age effect: \#MODEL AGE-PERIOD-COHORT model

\{ \#\#\# Fit of the Period, Cohort and age for (n in $1: \mathrm{N}$ ) \{

Y.ap [n] dpois (expected.Y.ap [n]);

\# Modeling rate

$\log ($ expected.Y.ap[n] $)<-\log ($ N.ap [n] ) +alpha [age[n] ] + beta [period [n] ] + gamma [cohort $[n]$ ]

\}

\#PERIOD: prior standard deviation

tau2.period<-K.s*pow (sigma.period, -2)

sigma.period dunif $(0.01,1)$;

\#\#\#\# PERIOD REF 1985-89

betamean [1] <- 0.0 ;

betaprec [1] <- tau2.period;

betamean [2] <- 0.0 ;

betaprec [2] <- tau2.period;

for $(j$ in $3: J)\{$

betamean $[j]<-2 * \operatorname{beta}[j-1]-\operatorname{beta}[j-2]$;

betaprec $[j]<-$ tau2 period;

\}

beta $[1]<-0$

for $(j$ in $2: J)\{$

beta $[j] \sim$ dnorm(betamean $[j]$, betaprec $[j])$;

\}

\#COHORT: prior standard deviation

tau2. cohort<-K. * $^{*}$ pow (sigma. cohort, -2 )

sigma.cohort dunif $(0.01,1)$;

\#\#\#\# PERIOD REF 1985-89

gammamean $[1]<-0.0$;

gammaprec [1] <- tau2. cohort;

gammamean [2] <- 0.0 ;

gammaprec [2] <- tau2. cohort;

for $(\mathrm{k}$ in $3: \mathrm{K})\{$

gammamean $[\mathrm{k}]<-2 *$ gamma $[\mathrm{k}-1]$ - gamma $[\mathrm{k}-2]$;

gammaprec $[\mathrm{k}]<-$ tau2. cohort;

\}

gamma $[1]<-0$

for $(\mathrm{k}$ in $2: \mathrm{K})\{$

gamma $[k] \sim$ dnorm (gammamean $[k]$, gammaprec $[k]$ );

\}

\#\#\#\# AGE

alphamean[1] <- 2*alpha[2] - alpha[3];

alphamean[2] <- (2*alpha[1] + 4*alpha[3] - alpha[4])/5;

for (i in $3:(I-2))$ \{

alphamean [i] <- (4*alpha[i-1] + 4*alpha[i+1]- alpha[i-2]

- alpha $[i+2]) / 6$;

\}

alphamean [I-1] <- (2*alpha[I] + 4*alpha[I-2] - alpha[I-3])/5;

Nneighsa $[I-1]<-5$;

alphamean [I] <- 2*alpha[I-1] - alpha[I-2];

for (i in $1: I)\{$

alphaprec $[i]<-$ tau2.age;

\}

for ( $i$ in $1: I)\{$

alpha [i] dnorm(alphamean [i], alphaprec [i]);

\}

tau2.age<-K.s*pow (sigma.age, -2 );

\#AGE: prior standard deviation

sigma.age dunif $(0.01,1)$;

\} 


\section{References}

[1] Ferlay J, Autier P, Boniol M, Heanue M, Colombet M, Boyle P. Estimates of the cancer incidence and mortality in Europe in 2006. Ann Oncol 2007;18(3):58192

[2] Bray F, Richiardi L, Ekbom A, Pukkala E, Cuninkova M, Moller H. Trends in testicular cancer incidence and mortality in 22 European countries: continuing increases in incidence and declines in mortality. Int J Cance 2006;118(12):3099-111.

[3] Huyghe E, Plante P, Thonneau PF. Testicular cancer variations in time and space in Europe. Eur Urol 2007;51(3):621-8.

[4] Richiardi L, Akre O, Lambe M, Granath F, Montgomery SM, Ekbom A. Birth order, sib ship size, and risk for germ-cell testicular cancer. Epidemiology 2004;15(3):323-9.

[5] Hsieh CC, Lambe M, Trichopoulos D, Ekbom A, Akre O, Adami HO. Early life exposure to oestrogen and testicular cancer risk: evidence against an aetiological hypothesis. Br J Cancer 2002;86(8):1363-4.

[6] Moller H, Jorgensen N, Forman D. Trends in incidence of testicular cancer in boys and adolescent men. Int J Cancer 1995;61(6):761-4.

[7] Akre O, Lipworth L, Cnattingius S, Sparen P, Ekbom A. Risk factor patterns for cryptorchidism and hypospadias. Epidemiology 1999;10(4):364-9.

[8] Pettersson A, Kaijser M, Richiardi L, Askling J, Ekbom A, Akre O. Women smoking and testicular cancer: one epidemic causing another? Int J Cancer 2004;109(6):941-4.

[9] Weir HK, Marrett LD, Kreiger N, Darlington GA, Sugar L. Pre-natal and perinatal exposures and risk of testicular germ-cell cancer. Int $\mathrm{J}$ Cancer 2000;87(3):438-43.

[10] Alfonso JL, Cortes C, Gimenez F, Cortina P. Cancer of the testis: risk factors and evolution of mortality in Spain (1951-1983). Arch Esp Urol 1989;42(8):75560.

[11] Fernandez QG, Monge JV, Lopez-Abente OG, Vincente Perez JA. Transitory trends in the mortality of testicular cancer in Spain 1953-1977. Actas Urol Esp 1988;12(4):340-6.

[12] Karim-Kos HE, de Vries E, Soerjomataram I, Lemmens V, Siesling S, Coebergh JW. Recent trends of cancer in Europe: a combined approach of incidence, survival and mortality for 17 cancer sites since the. Eur J Cancer 1990;2008.

[13] Spiegelhalter DJ, Thomas A, Best N, Lunn D. WinBUGS User Manual, Version 1.4. Cambridge, England: MRC Biostatistics Unit, 2002.

[14] R Development Core Team. R: A language and environment for statistica computing, R Foundation for Statistical Computing, Vienna, Austria, 2006 ISBN 3-900051-07-0. http://www.R-project.org 2006; Available at: URL: http://www.R-project.org.

[15] Sturtz S, Ligges U, Gelman A. R2WinBUGS: a package for running WinBUGS from R. J Stat Soft 2005;12(3):1-16.

[16] World Health Organization. International classification of diseases, Tenth Revision (ICD-10). Geneva: WHO, 1992.

[17] Clayton D, Schifflers E. Models for temporal variation in cancer rates. II. Ageperiod-cohort models. Stat Med 1987;6(4):469-81.

[18] Bashir SA, Estève J. Projecting cancer incidence and mortality using Bayesian age-period. Cohort models. J Epidemiol Biostat 2001;6:287-96.

[19] Knorr-Held L, Rainer E. Projections of lung cancer mortality in West Germany: a case study in Bayesian prediction. Biostatistics 2001;2(1):109-29.

[20] Cleries R, Ribes J, Esteban L, Martinez JM, Borras JM. Time trends of breast cancer mortality in Spain during the period 1977-2001 and Bayesian approach for projections during 2002-2016. Ann Oncol 2006;17(12):1783-91.

[21] Gelman A. Prior distributions for variance parameters in hierarchical models. Bayesian Anal 2005;1:1-19.

[22] Spiegelhalter DJ, Best NG, Carlin BP, van der Linde AJ, Bayesian. Measures of model complexity and fit (with discussion). J Roy Stat Soc Ser B 2002;64(3):583-639.
[23] Holford TR. The estimation of age, period and cohort effects for vital rates. Biometrics 1983;39:311-24.

[24] Holford TR. Understanding the effects of age, period, and cohort on incidence and mortality rates. Annu Rev Publ Health 1991;12:425-57.

[25] Holford TR. An alternative approach to statistical age-period-cohort analysis. J Chronic Dis 1985;38(10):831-40.

[26] Holford TR. Analysing the temporal effects of age, period and cohort. Stat Methods Med Res 1992;1(3):317-37.

[27] Einhorn LH, Donohue J. Cis-diamminedichloroplatinum, vinblastine, and bleomycin combination chemotherapy in disseminated testicular cancer. Ann Intern Med 1977;87(3):293-8.

[28] Becker N, Boyle P. Decline in mortality from testicular cancer in West Germany after reunification. Lancet 1997;350(9079):744.

[29] Thornhill JA, Conroy RM, Kelly DG, Walsh A, Fennelly JJ, Fitzpatrick JM. Recent trends in mortality due to testicular cancer in Ireland: a comparison with England and Wales. J Epidemiol Community Health 1986;40(3):218-22.

[30] Brown LM, Pottern LM, Hoover RN, Devesa SS, Aselton P, Flannery JT. Testicular cancer in the United States: trends in incidence and mortality. Int J Epidemiol 1986;15(2):164-70.

[31] Osterlind A. Diverging trends in incidence and mortality of testicular cancer in Denmark, 1943-1982. Br J Cancer 1986;53(4):501-5.

[32] Richiardi L, Bellocco R, Adami HO, Torrang A, Barlow L, Hakulinen T, et al. Testicular cancer incidence in eight northern European countries: secular and recent trends. Cancer Epidemiol Biomarkers Prev 2004;13:2157-66.

[33] Peckham MJ, McElwain TJ, Barrett A, Hendry WF. Combined management of malignant teratoma of the testis. Lancet 1979;2(8137):267-70.

[34] Oliver RT, Ong J, Blandy JP, Altman DG. Testis conservation studies in germ cell cancer justified by improved primary chemotherapy response and reduced delay, 1978-1994. Br J Urol 1996;78(1):119-24.

[35] Cambil J. Effectiveness of testicular self-examination and screening for an early detection of testicular cancer. Index Enferm 2005;14:48-9.

[36] Webb V, Holmes A. Urological cancers: do early detection strategies exist? BJU Int 2000;86(9):996-1000.

[37] Sant M, Aareleid T, Artioli ME, Berrino F, Coebergh JW, Colonna M, et al. Tenyear survival and risk of relapse for testicular cancer: a EUROCARE high resolution study. Eur J Cancer 2007;43(3):585-92.

[38] Einhorn LH. Curing metastatic testicular cancer. Proc Natl Acad Sci USA 2002;99(7):4592-5.

[39] Sant M, Aareleid T, Berrino F, Lasota MB, Carli PM, Faivre J, et al. EUROCARE-3: survival of cancer patients diagnosed 1990-94-results and commentary. Ann Oncol 2003:14(Suppl. 5):v61-118.

[40] Berrino F, De Angelis R, Sant M, Rosso S, Lasota MB, Coebergh JW, et al. Survival for eight major cancers and all cancers combined for European adults diagnosed in 1995-99: results of the EUROCARE-4 study. Lancet Oncol 2007;8(9):773-83.

[41] Berrino F, Capocaccia R, Estève J, Gatta G, Hakulinen T, Micheli A, et al. Survival of cancer patients in Europe: the EUROCARE-2 Study. IARC Scientific Press; 1999.

[42] Eurostat. Non-national populations in the EU Member States. Eurostat Population and social conditions 2006; Issue number 8; Available at: URL: http:// epp.eurostat.ec.europa.eu/cache/ITY_OFFPUB/KS-NK-06-008/EN/KS-NK-06008-EN.PDF, Accessed on June 15, 2006.

[43] Bray F, Moller B. Predicting the future burden of cancer. Nat Rev Cancer 2006;6(1):63-74.

[44] Richie JP. Impact of diagnostic delay in testis cancer: results of a large population-based study Huyghe E, Muller A, Mieusset R, Bujan L, Bachaud JM, Chevreau C, Plante P, Thonneau P, Human Fertility Research Group, Paule de Viguier Hospital, Toulouse University III, France; Urology and Andrology Department, Paule de Viguier Hospital, Toulouse, France. Urol Oncol $2008 ; 26(2): 220-1$ 